\title{
La independencia de una versión débil de la conjetura del espacio normal de Moore
}

\author{
Carlos Mario Parra-Londoño*, Andrés Felipe Uribe-Zapata \\ Universidad Nacional de Colombia, Escuela de Matemáticas, Medellín, Colombia.
}

\begin{abstract}
Resumen. Nuestro propósito es presentar una exposición elemental de un resultado clásico en topología general, que es una versión débil de un problema conocido como la conjetura del espacio normal de Moore. Con este fin, se definen y estudian algunas propiedades básicas de los espacios de Moore y se caracterizan aquellos que a su vez son segundo contables y de Lindelöf. Además, se enuncia la hipótesis del continuo y el Axioma de Martin, y se aplican para establecer la independencia del problema en cuestión.

Palabras clave: Espacio de Moore, independencia, hipótesis del continuo, Axioma de Martin, $Q$-set.

MSC2010: 03E35, 03E50, 03E65, 54E30, 54E35.

\section{The independence of a weak version of the normal Moore space conjecture}

\begin{abstract}
Our purpose is to present an elementary exposition of a classical result in general topology which is a weak version of a problem known as the normal Moore space conjecture. With this aim we study some of the basic properties of Moore spaces and characterize those which are both Lindelof and second countable. We also make use of the continuum hypothesis along with Martin's axiom to establish the result in question.

Keywords: Moore's space, independence, continuum hypothesis, Martin's Axiom, $Q$-set.
\end{abstract}

\section{Introducción}

Desde el trabajo de K. Gödel se sabe que existen afirmaciones que no se pueden decidir en la teoría de conjuntos de Zermelo-Fraenkel (ZFC). Entre dichas afirmaciones se destaca

\footnotetext{
*E-mail: cmparra@unal.edu.co

Recibido: 3 de septiembre de 2019, Aceptado: 1 de noviembre de 2019.

Para citar este articulo: C.M. Parra-Londoño y A.F. Uribe-Zapata, La independencia de una versión dé-

bil de la conjetura del espacio normal de Moore, Rev. Integr. temas mat. 38 (2020), No. 1, 43-54. doi: 10.18273/revint.v38n1-2020004
} 
especialmente la hipótesis del continuo $(\mathbf{C H})$ cuya independencia fue establecida en parte por Gödel en su tiempo, y definitivamente por Paul Cohen en 1963.

En este artículo se discute la independencia de una afirmación en topología general que llamamos la conjetura débil del espacio normal de Moore (MWC), la cual afirma que todo espacio normal de Moore separable es metrizable. Dicha conjetura fue propuesta por F. B. Jones en los años 30 y su independencia es consecuencia del trabajo conjunto del mismo Jones, F. D. Tall, J. H. Silver y R. H Bing (véase [1], [3] y [6]). En la tesis doctoral de F. D. Tall (remitirse a [6]) se bosqueja la demostración de la consistencia de $\neg$ MWC; sin embargo, algunos resultados claves aparecen sin demostración. Por otro lado, la consistencia de MWC se resuelve en el artículo de F.B Jones (véase [3]); no obstante, sus demostraciones son muy complicadas, y por tanto presentamos pruebas detalladas más simples y en un lenguaje actual.

La conjetura débil del espacio normal de Moore es un caso especial de un problema mucho más difícil que por décadas ha sido central en topología general: la conjetura del espacio normal de Moore ${ }^{1}$, cuya solución se relaciona con nociones avanzadas en teoría de conjuntos tales como forzamiento, grandes cardinales, modelos internos, entre otras. En la década de los 80 se demostró que dicho problema involucra la existencia de cierto tipo de gran cardinal llamado "cardinal medible" (véase [2] y [5]), lo cual sitúa la conjetura en el marco de la teoría de grandes cardinales.

Al igual que con la conjetura del espacio normal de Moore, las demostraciones típicas de independencia requieren un conocimiento avanzado en teoría de conjuntos, lógica matemática y el área específica del problema. De manera que el propósito de este artículo es ilustrar una demostración de independencia al alcance de un estudiante de pregrado con conocimientos básicos en topología general y teoría de conjuntos. Si bien en la primera parte se introducen formalmente la hipótesis del continuo y el Axioma de Martin, el lector puede prescindir de ellos y solo considerar las consecuencias consignadas en los Teoremas 2.3 y 2.10 , las cuales para nuestros objetivos pueden pensarse como axiomas.

A lo largo del texto todos los espacios se consideran $T_{1}$; por una vecindad en un punto se entiende un abierto que lo contiene, y se denota por $\omega$ el conjunto de los números naturales.

\section{Nociones de lógica y teoría de conjuntos}

Se dice que un conjunto de sentencias $\Sigma$ es consistente si de él no se puede deducir una contradicción. Si $\varphi$ es una sentencia, entonces $\varphi$ es consistente con $\Sigma$, si $\Sigma+\varphi:=\Sigma \cup\{\varphi\}$ es consistente. Ahora bien, $\varphi$ es independiente de $\Sigma$ si tanto $\varphi$ como $\neg \varphi$ son consistentes con $\Sigma$, lo cual indica que $\varphi$ no se puede demostrar ni refutar en $\Sigma$. A continuación se enuncia un resultado elemental de lógica, que permitirá realizar las demostraciones de consistencia:

Lema 2.1. Sean $\Sigma$ un conjunto de sentencias y $\varphi$ una sentencia. Si $\Sigma \vdash(\varphi \Longrightarrow \psi)$ y $\varphi$ es consistente con $\Sigma$, entonces $\psi$ es consistente con $\Sigma$.

De manera que, para demostrar que cierta sentencia $\psi$ es consistente con ZFC, bastará con hallar otra sentencia $\varphi$ consistente con ZFC, tal que $\varphi$ implique $\psi$. En nuestro caso,

\footnotetext{
${ }^{1}$ La cual afirma que todo espacio normal de Moore es metrizable.
} 
dicha sentencia $\varphi$ es la hipótesis del continuo por un lado y el Axioma de Martin por el otro, los cuales se introducen a continuación:

\subsection{La hipótesis del continuo}

Se denota por $\aleph_{0}$ al cardinal de los números naturales y por $\aleph_{1}$ al mínimo cardinal no contable. Dado un conjunto $A$, se denota por $2^{|A|}$ al cardinal de partes de $A, \mathcal{P}(A)$. La hipótesis del continuo $(\mathbf{C H})$ es la sentencia $2^{\aleph_{0}}=\aleph_{1}$, lo cual equivale a que no existen subconjuntos de números reales cuyo cardinal esté estrictamente entre $\aleph_{0}$ y el cardinal de $\mathbb{R}$. Esta hipótesis planteada por Georg Cantor fue uno de los grandes problemas de la matemática en el siglo pasado, al punto de ser incluido por David Hilbert como el primero de su famosa lista de 23. En 1939, K. Gödel demostró su consistencia (remitirse a $[4$, p. 165$])$ :

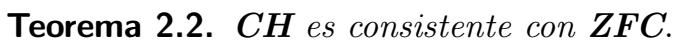

Para nuestros propósitos solo será necesaria una consecuencia inmediata de $\mathbf{C H}$ y del Teorema de Cantor:

Teorema 2.3. La hipótesis del continuo implica que $2^{\aleph_{0}}<2^{\aleph_{1}}$.

\subsection{El Axioma de Martin}

El Axioma de Martin involucra conjuntos parcialmente ordenados y afirma la existencia de ciertos subconjuntos denominados "genéricos" (véase la Definición 2.5). Es una consecuencia inmediata de $\mathbf{C H}$, y por tanto es consistente con ZFC. Para enunciarlo se debe introducir la siguiente terminología:

Definición 2.4. Una relación $\leq$ sobre un conjunto $P$ es un preorden si es reflexiva y transitiva. Al par $(P, \leq)$ se lo llama conjunto preordenado. Ahora bien, dado un conjunto preordenado $(P, \leq)$, se definen:

1. $p, q \in P$ son compatibles, si existe $r \in P$ tal que $r \leq p$ y $r \leq q$.

2. Dos elementos de $P$ son incompatibles si no son compatibles.

3. $A \subseteq P$ es una anticadena si cualquier par de elementos de $A$ son incompatibles.

4. $D \subseteq P$ es denso si para todo $p \in P$, existe $d \in D$ tal que $d \leq p$.

5. Se dice que $P$ satisface la condición de cadena contable (ccc), si toda anticadena es a lo sumo contable.

Definición 2.5. Sean $(P, \leq)$ un conjunto preordenado y $D \subseteq P$ denso. Entonces se dice que $G \subseteq P$ es un filtro $D$-genérico si satisface las siguientes condiciones:

1. G es cerrado hacia arriba, es decir, si $a \in G$ y $a \leq b$, entonces $b \in G$.

2. Todo par de elementos de $G$ son compatibles mediante un elemento de $G$.

3. $D \cap G \neq \emptyset$.

Vol. 38, $\left.\mathrm{N}^{\circ} 1,2020\right]$ 
Si $\mathcal{D}$ es una colección de subconjuntos densos de $P$, entonces $G$ es un filtro $\mathcal{D}$-genérico si es un filtro $D$-genérico para todo $D \in \mathcal{D}$.

Particularmente cuando la colección $\mathcal{D}$ es contable, se puede garantizar la existencia de filtros $\mathcal{D}$-genéricos mediante el siguiente resultado:

Teorema 2.6. Sean $(P, \leq)$ un conjunto preordenado y $\mathcal{D}$ una colección contable de subconjuntos densos de $P$. Entonces existe un filtro $\mathcal{D}$-genérico en $P$.

Demostración. Sean $p \in P$ y $\mathcal{D}:=\left\{D_{n}: n \in \omega\right\}$ una colección contable de subconjuntos densos de $P$. Defínase por recursión sobre $\omega$ una sucesión de puntos de $P,\left\{r_{n}\right\}_{n \in \omega}$, tal que $r_{0}:=p$ y para $n \in \omega, r_{n+1}$ es una extensión de $r_{n}$ en $D_{n}$, lo cual es posible ya que $D_{n}$ es denso. Ahora bien, considérese $G:=\left\{q \in P:(\exists n \in \omega)\left(r_{n} \leq q\right)\right\}$. Se afirma que $G$ es un filtro $\mathcal{D}$-genérico. En efecto, es inmediato que $G$ es cerrado hacia arriba. Por otro lado, como $\left\{r_{n}\right\}_{n \in \omega}$ es una sucesión decreciente, se tiene que todo par de elementos son compatibles mediante un $r_{i}$ y además, para cada $n \in \omega, r_{n+1} \in D_{n} \cap G$, de modo que $G$ interseca cada elemento de $\mathcal{D}$. Finalmente, $G$ es un filtro $\mathcal{D}$-genérico.

Resulta natural entonces preguntarse por la existencia de filtros $\mathcal{D}$-genéricos cuando $\mathcal{D}$ es no contable.

Definición 2.7. Se denota por MA $(\kappa)$ la afirmación: "Si $\kappa$ es un cardinal infinito, $(P, \leq)$ es un conjunto preordenado que satisface la ccc y $\mathcal{D}$ es una colección de a lo sumo $\kappa$ subconjuntos densos de $P$, entonces existe un filtro $\mathcal{D}$-genérico en $P$ ".

Si el Teorema 2.6 se aplica a conjuntos preordenados que satisfacen la ccc se sigue inmediatamente que MA $\left(\aleph_{0}\right)$ es cierto. Por otro lado, se puede demostrar que si $\kappa \geq 2^{\aleph_{0}}$, entonces $\mathbf{M A}(\kappa)$ es falso (véase [4, p. 54]). De manera que, solo restaría determinar $\mathbf{M A}(\kappa)$ cuando $\aleph_{0}<\kappa<2^{\aleph_{0}}$.

Definición 2.8. Se define como Axioma de Martin (MA) la sentencia: "Para todo cardinal $\kappa$, si $\aleph_{0} \leq \kappa<2^{\aleph_{0}}$, entonces $\mathbf{M A}(\kappa)$ es cierto".

Si se supone $\mathbf{C H}$ entonces el único cardinal infinito menor que $2^{\aleph_{0}}$ es precisamente $\aleph_{0}$, de manera que MA se reduce a MA $\left(\aleph_{0}\right)$, el cual es cierto en virtud del Teorema 2.6. Por consiguiente, la hipótesis del continuo trivializa el Axioma de Martin y por tanto es usual usarlo suponiendo además su negación, lo cual es consistente con ZFC (remitirse a $[4$, p. 278]):

Teorema 2.9. $\boldsymbol{M A}+\neg \boldsymbol{C H}$ es consistente con $\boldsymbol{Z F C}$.

Un aplicación típica del Axioma de Martin tiene que ver con las propiedades de las familias casi disjuntas de $\omega$ : una familia de conjuntos infinitos $\mathcal{F} \subseteq \mathcal{P}(\omega)$ se dice casi disjunta, si cualquier par de elementos de $\mathcal{F}$ tienen intersección finita. Este tipo de familias jugarán un papel fundamental en nuestro desarrollo mediante el siguiente resultado (véase [4, p. 57]):

Teorema 2.10. Considérese $\mathcal{A} \subseteq \mathcal{P}(\omega)$ una familia casi disjunta de cardinal $\kappa$ donde $\aleph_{0} \leq \kappa<2^{\aleph_{0}}$ y $\mathcal{B} \subseteq \mathcal{A}$. Si $\boldsymbol{M A}(\kappa)$ es cierto, entonces existe $c \subseteq \omega$ tal que para todo $x \in \mathcal{B},|x \cap c|<\aleph_{0}$, y para todo $x \in \mathcal{A} \backslash \mathcal{B},|x \cap c|=\aleph_{0}$.

[Revista Integración, temas de matemáticas 


\section{Espacios de Moore}

\subsection{Desarrollos}

El topólogo R. L. Moore en los años 20 introdujo la noción de desarrollo ${ }^{2}$, la cual se puede pensar como un axioma de enumerabilidad:

Definición 3.1. Un desarrollo sobre un espacio topológico $X$ es una colección contable $\left\{\mathcal{U}_{n}\right\}_{n \in \omega}$ de cubrimientos abiertos de $X$ tal que para todo $x \in X$ y toda vecindad $U$ de $x$, existe $N \in \omega$ el cual satisface que para todo $V \in \mathcal{U}_{N}$, si $x \in V$ entonces $V \subseteq U$.

Definición 3.2. Un espacio topológico es un espacio de Moore si es regular y tiene un un desarrollo.

\subsection{Propiedades básicas}

Teorema 3.3. Todo espacio de Moore es primero contable.

Demostración. Sean $M$ un espacio de Moore, $\left\{\mathcal{U}_{n}\right\}_{n \in \omega}$ un desarrollo sobre $M$ y $x \in M$. Consíderese para todo $n \in \omega, U_{n} \in \mathcal{U}_{n}$ tal que $x \in U_{n}$. Lo anterior es posible ya que $\mathcal{U}_{n}$ cubre a $X$. Se afirma que $\left\{U_{n}\right\}_{n \in \omega}$ es una base local contable en $x$. En efecto, sea $U$ una vecindad de $x$. Entonces existe $N \in \omega$ tal que para toda vecindad $V$ de $x$, si $V \in \mathcal{U}_{N}$ entonces $V \subseteq U$. Como $x \in U_{N}$ y $U_{N} \in \mathcal{U}_{N}$, entonces $U_{N} \subseteq U$. Luego $M$ es primero contable.

Teorema 3.4. Todo espacio métrico es un espacio de Moore.

Demostración. Sea $(X, d)$ un espacio métrico. $(\forall n \in \omega)\left(\mathcal{U}_{n}:=\left\{B\left(x, \frac{1}{n}\right): x \in X\right\}\right)$. Se afirma que $\left\{\mathcal{U}_{n}\right\}_{n \in \omega}$ es un desarrollo sobre $X$. En efecto, es claro que es una colección contable de cubrimientos abiertos de $X$. Ahora bien, dados $x \in X$ y $U$ una vecindad de $x$, existe $N \in \omega$ tal que $B\left(x, \frac{1}{N}\right) \subseteq U$. Sea $V \in \mathcal{U}_{2 N}$ tal que $x \in V$. Entonces $V=B\left(y, \frac{1}{2 N}\right)$, donde $y \in X$ y $d(x, y)<\frac{1}{2 N}$. Nótese que si $z \in V$, entonces:

$$
d(x, z) \leq d(x, y)+d(y, z)<\frac{1}{2 N}+\frac{1}{2 N}=\frac{1}{N}
$$

de donde $z \in B\left(x, \frac{1}{N}\right) \subseteq U$ y por tanto, $V \subseteq U$. Finalmente, $X$ es regular y tiene un desarrollo, es decir, es un espacio de Moore.

El recíproco del Teorema anterior no es cierto: existen espacios de Moore que no son metrizables (remitirse al Teorema 4.9). Más aún, existen espacios de Moore que no son normales (véase el comentario posterior al Teorema 4.11).

\footnotetext{
${ }^{2}$ Del inglés development.
}

Vol. 38, $\mathrm{N}^{\circ} 1,2020$ ] 


\section{3. ¿Espacios métricos?}

En esta sección se busca motivar de forma natural la conjetura del espacio normal de Moore, exponiendo propiedades en común entre los espacios normales de Moore y los espacios métricos.

A continuación se introduce, por simplicidad, una notación que se adoptará en adelante:

Definición 3.5. Un espacio topológico $X$ es Jones-compacto si todo subconjunto no contable de $X$ tiene un punto de acumulación.

Teorema 3.6. Todo espacio de Lindelöf es Jones-compacto.

Demostración. Sea $X$ un espacio de Lindelöf. Razonando por contradicción, sea $Y \subseteq X$, no contable y sin puntos de acumulación. En consecuencia, $Y$ es cerrado y por tanto $X \backslash Y$ es abierto. Además, para todo $y \in Y$, existe un abierto $U_{y}$, tal que $U_{y} \cap Y=\{y\}$. Nótese que $\bigcup_{y \in Y} U_{y} \cup(X \backslash Y)$ es un cubrimiento abierto de $X$ y por tanto, como $X$ es de Lindelöf, puede ser cubierto por una cantidad contable de dichos conjuntos. Por otro lado, ya que $X \backslash Y$ no interseca a $Y$ y cada vecindad $U_{y}$ contiene un único punto de $Y$, entonces $Y$ debe ser contable, lo cual es absurdo. Finalmente, $X$ es Jones-compacto. $\square$

En seguida se expone una condición para que un espacio de Moore sea de Lindelöf:

Teorema 3.7 (F. B Jones, 1933). Todo espacio Jones-compacto y de Moore es un espacio de Lindelöf.

Demostración. Sean $M$ un espacio de Moore y Jones-compacto, $\left\{\mathcal{U}_{n}\right\}_{n \in \omega}$ un desarrollo sobre $M$ y $\mathcal{C}$ un cubrimiento abierto de $M$. Por el Teorema de buen orden, $M$ se puede escribir en la forma $M=\left\{m_{\beta}: \beta<\alpha\right\}$, donde $\alpha$ es un ordinal. Fíjese $n \in \omega$. Se define, por recursión transfinita sobre $\alpha$, una función $p_{n}: \alpha \rightarrow M$ como sigue:

1. $p_{n}(0)$ es el mínimo elemento de $M$ para el cual existe $C_{0} \in \mathcal{C}$ tal que si $U \in \mathcal{U}_{n}$ y $p_{n}(0) \in U$, entonces $U \subseteq C_{0}$, si dicho mínimo existe. De lo contrario, $p_{n}(0):=m_{0}$ y $C_{0} \in \mathcal{C}$ es un abierto arbitrario.

2. $p_{n}(\gamma)$ es el mínimo elemento de $M \backslash \bigcup_{\beta<\gamma} C_{\beta}$ para el cual existe $C_{\gamma} \in \mathcal{C}$ tal que si $U \in \mathcal{U}_{n}$ y $p_{n}(\gamma) \in U$, entonces $U \subseteq C_{\gamma}$, si dicho mínimo existe. De lo contrario, $p_{n}(\gamma):=m_{0}$ y $C_{\gamma}:=C_{0}$.

Nótese que $\left\{p_{n}(\beta): \beta<\alpha\right\}$ es contable, ya que si no lo fuese, entonces por hipótesis tendría un punto de acumulación $p$. Sean $U \in \mathcal{U}_{n}$ tal que $p \in U$ y $p_{n}(\gamma), p_{n}(\lambda) \in U$ distintos. Así pues, $p_{n}(\gamma), p_{n}(\lambda) \in U$ y por tanto, $U \subseteq C_{\gamma}$ y $U \subseteq C_{\lambda}$. De donde $p_{n}(\gamma) \in$ $C_{\lambda}$ y $p_{n}(\lambda) \in C_{\gamma}$ lo cual es absurdo por como se definió la recursión. De manera que $\mathcal{D}_{n}:=\left\{C_{\beta}: \beta<\alpha\right\}$ es una subcolección contable de $\mathcal{C}$. Por otro lado, obsérvese que si el mínimo de la definición recursiva no existe, entonces el recorrido de la función $p_{n}$ es $\left\{m_{0}\right\}$, es decir, la función colapsa en $m_{0}$. Además, es claro de la definición que mientras la función no colapse, se satisface que para todo $\beta<\alpha, m_{\beta} \leq p_{n}(\beta)$.

Ahora bien, se define $\mathcal{D}:=\bigcup_{n \in \omega} \mathcal{D}_{n}$ y se afirma que es un subcubrimiento contable de $\mathcal{C}$. En efecto, para ver que cubre a $M$, razonando por contradicción, supóngase que existe

[Revista Integración, temas de matemáticas 
$x \in M$ tal que $x \notin \mathcal{D}$, y sea $C \in \mathcal{C}$ tal que $x \in C$. Como $M$ es un espacio de Moore, existe $N \in \omega$ tal que para todo $U \in \mathcal{U}_{N}$, si $x \in U$, entonces $U \subseteq C$. Además, ya que $x \in M$, existe $\lambda \in \alpha$ tal que $x=m_{\lambda}$. Obsérvese que $m_{\lambda}$ satisface la definición de la recursión, y por tanto la función $p_{N}$ no ha colapsado hasta el valor de $\lambda$, de manera que $m_{\lambda} \leq p_{N}(\lambda)$ y, por consiguiente, $x=m_{\lambda} \leq p_{N}(\lambda) \leq x$, de donde $x=p_{N}(\lambda)$, lo cual es una contradicción, ya que $x=p_{N}(\lambda) \in C_{\lambda} \in \mathcal{D}_{N}$. Finalmente, $\mathcal{D}$ es subcubrimiento contable de $\mathcal{C}$ y por tanto, $M$ es un espacio de Lindelöf.

Como resultado de los dos teoremas anteriores, se obtiene la siguiente caracterización:

Teorema 3.8. Un espacio de Moore es de Lindelöf si, y solo si, es Jones-compacto.

Ahora se expone una condición suficiente para que un espacio de Moore sea segundo contable.

Teorema 3.9. Todo espacio de Moore y de Lindelöf es segundo contable.

Demostración. Sean $M$ un espacio de Moore y de Lindelöf y $\left\{\mathcal{U}_{n}\right\}_{n \in \omega}$ un desarrollo sobre $M$. Para todo $n \in \omega$, denótese por $\mathcal{B}_{n}$ un subcubrimiento contable obtenido de $\mathcal{U}_{n}$ y considérese $\mathcal{B}:=\bigcup_{n \in \omega} \mathcal{B}_{n}$. Se afirma que $\mathcal{B}$ es una base contable. En efecto, es contable ya que cada $\mathcal{B}_{n}$ se escogió contable. Para ver que es una base, sean $U \subseteq M$ abierto y $x \in U$. Así que, existe $N \in \omega$ tal que para toda vecindad $V$ de $x$, si $V \in \mathcal{U}_{N}$, entonces $V \subseteq U$. Como particularmente $\mathcal{B}_{N}$ cubre a $M$, existe un abierto $V \in \mathcal{B}_{N} \subseteq \mathcal{U}_{N}$ tal que $x \in V$, y por tanto $x \in V \subseteq U$. Finalmente, $\mathcal{B}$ es una base contable.

Corolario 3.10. Si $M$ es un espacio de Moore y Jones-compacto, entonces es segundo contable.

De manera que según los resultados anteriores, se obtiene una caracterización de los espacios de Moore que son segundo contables, de Lindelöf y Jones-compactos:

Teorema 3.11. En un espacio de Moore las siguientes propiedades son equivalentes: es segundo contable, de Lindelöf y Jones-compacto.

En resumen, algunas de las propiedades que satisfacen los espacios métricos y los espacios normales de Moore son: la equivalencia entre segundo contable y de Lindelöf, primero contable, regular, normal y además completamente normal (véase [3, p. 676]). De manera que la pregunta ¿será que todo espacio normal de Moore es métrico? aparece naturalmente de las propiedades expuestas ${ }^{3}$. No obstante, como se establecerá en la siguiente sección, dicha pregunta resulta ir más allá de los límites de ZFC. Como diría Paul Erdös: el monstruo feo de la incompletez aparece de nuevo y en "lugares" inesperados como la topología general.

\section{La independencia}

En esta sección, con base en la consistencia de $\mathbf{C H}$ y $\mathbf{M A}+\neg \mathbf{C H}$, se demuestra detalladamente la independencia de MWC.

\footnotetext{
${ }^{3}$ Más aún, cuando se añade la hipótesis de separabilidad del espacio.
} 


\subsection{Consistencia}

Se empieza por demostrar la consistencia. La idea básica es la siguiente: ya se ha demostrado que ser Jones-compacto es equivalente a ser segundo contable en un espacio de Moore, y que esto es suficiente para concluir la metrizabilidad, ya que el espacio se supone normal. De modo que solo resta establecer condiciones para que un espacio normal de Moore sea Jones-compacto. Aquí es donde la hipótesis de separabilidad y $2^{\aleph_{0}}<2^{\aleph_{1}}$ juegan su papel:

Teorema 4.1 (F. B. Jones, 1933). Si $2^{\aleph_{1}}>2^{\aleph_{0}}$, entonces todo espacio normal y separable es Jones-compacto.

Demostración. Supóngase que $2^{\aleph_{0}}<2^{\aleph_{1}}$. Sean $X$ un espacio normal y separable y $D \subseteq X$ denso y contable. Razonando por contradicción, supóngase que existe $Y \subseteq X$ no contable y sin puntos de acumulación. Así que $Y$ es cerrado, más aún, todo subconjunto de $Y$ es cerrado. Sea $A$ un subconjunto propio no vacío de $Y$; entonces $A$ y $Y \backslash A$ son cerrados y disjuntos, así que por la normalidad existen abiertos disjuntos $U_{A}$ y $V_{A}$ tales que $A \subseteq U_{A}$ y $Y \backslash A \subseteq V_{A}$. A continuación, considérese la función $\varphi: \mathcal{P}(Y) \rightarrow \mathcal{P}(D)$ definida como sigue: $\varphi(\emptyset):=\emptyset, \varphi(Y):=D$ y si $\emptyset \subsetneq A \subsetneq Y$, entonces $\varphi(A):=D \cap U_{A}$. Se afirma que $\varphi$ es inyectiva. En efecto, sea $A$ un subconjunto propio no vacío de $Y$. Entonces $\varphi(A)=D \cap U_{A} \neq \emptyset$, puesto que $U_{A}$ es abierto y $D$ es denso. Además, $\varphi(A) \neq D$, ya que $D \cap V_{A}$ es no vacío. Ahora bien, sea $B$ subconjunto propio no vacío de $Y$ tal que $A \neq B$. Así pues, sin pérdida de generalidad existe $x \in A$ tal que $x \notin B$. De modo que $x \in Y \backslash B$ y por tanto, $x \in U_{A} \cap V_{B}$. Como este último conjunto es abierto y no vacío, debe contener puntos de $D$, a saber $y \in D \cap\left(U_{A} \cap V_{B}\right)$. En consecuencia, $y \in U_{A}$, y como $y \in V_{B}$, entonces $y \notin U_{B}$, de donde $y \in D \cap U_{A}$ y $y \notin D \cap U_{B}$. Lo anterior implica que $D \cap U_{A} \neq D \cap U_{B}$, es decir, $\varphi(A) \neq \varphi(B)$, y por tanto $\varphi$ es inyectiva.

Para concluir, sea $\kappa:=|Y|$. Ya que $Y$ es no contable, entonces $\aleph_{1} \leq \kappa$ y por tanto $2^{\aleph_{1}} \leq 2^{\kappa}$. Además, como $\varphi$ es inyectiva, $2^{\kappa} \leq 2^{\aleph_{0}}$. De manera que $2^{\aleph_{1}} \leq 2^{\kappa} \leq 2^{\aleph_{0}}$ y por consiguiente $2^{\aleph_{1}} \leq 2^{\aleph_{0}}$, lo cual contradice la hipótesis. Luego $X$ es Jones-compacto. $\square$

Corolario 4.2. $2^{\aleph_{0}}<2^{\aleph_{1}}$ implica $\boldsymbol{M W C}$.

Demostración. Supóngase que $2^{\aleph_{0}}<2^{\aleph_{1}}$ y sea $M$ un espacio normal y separable de Moore. Por el resultado anterior, $M$ es Jones-compacto, y por el Teorema $3.11 M$ es segundo contable, de manera que $M$ es segundo contable y normal. Finalmente, por el Teorema de metrización de Urysohn, $M$ es metrizable.

De modo que $\mathbf{C H}$ implica MWC, y como $\mathbf{C H}$ es consistente, entonces aplicando el Lema 2.1 resulta el

Corolario 4.3. $\boldsymbol{M W C}$ es consistente con $\boldsymbol{Z F C}$.

\subsection{Consistencia de la negación}

Ahora se aborda la consistencia de $\neg \mathbf{M W C}$. La idea es que de MA $+\neg \mathbf{C H}$ se sigue la existencia de cierto tipo de subconjunto de la recta real, lo cual a su vez implica la existencia de un espacio normal de Moore separable que no es metrizable.

[Revista Integración, temas de matemáticas 


\section{Q-sets}

Definición 4.4. Dado un espacio topológico $X$, se dice que $Y \subseteq X$ es un $\mathbf{F}_{\sigma}$, si $Y$ es una unión contable de subconjuntos cerrados.

Los subconjuntos mencionados al iniciar esta sección son los llamados $Q$-sets, los cuales se definen a continuación:

Definición 4.5. Sea $X \subseteq \mathbb{R}$ no contable. Entonces $X$ es un $Q$-set si todo subespacio de $X$ es un $F_{\sigma}$ en $X$.

Ahora se verá que la existencia de $Q$-sets se sigue de $\mathbf{M A}+\neg \mathbf{C H}$. Sin embargo, primero debemos considerar el siguiente Lema:

Lema 4.6. Existe una subcolección de $\mathcal{P}(\omega)$ casi disjunta cuyo cardinal es $2^{\aleph_{0}}$.

Demostración. Para todo $r \in \mathbb{R}$, existe una sucesión $\left\{a_{n}^{(r)}\right\}_{n \in \omega}$ de números racionales que converge a $r$. Sea $f: \omega \rightarrow \mathbb{Q}$ una función biyectiva, y para todo $r \in \mathbb{R}$ se define $A_{r}:=\left\{k \in \omega:(\exists n \in \omega)\left(f(k)=a_{n}^{(r)}\right)\right\}$. Entonces $\mathcal{A}:=\left\{A_{r}: r \in \mathbb{R}\right\}$ es una familia casi disjunta cuyo cardinal es $2^{\aleph_{0}}$.

Teorema 4.7 (J. H. Silver, 1969). $\boldsymbol{M A}+\neg \boldsymbol{C H}$ implica la existencia de un $Q$-set.

Demostración. Supóngase MA $+\neg \mathbf{C H}$. Por el Lema anterior y por $\neg \mathbf{C H}$, existe una subcolección casi disjunta de $\mathcal{P}(\omega)$ cuyo cardinal es $\aleph_{1}$, digamos, $\mathcal{A}=\left\{A_{\alpha}\right\}_{\alpha<\aleph_{1}}$. Ahora bien, ya que MA es cierto y $\aleph_{0}<\aleph_{1}<2^{\aleph_{0}}$, entonces se tiene $\mathbf{M A}\left(\aleph_{1}\right)$, de manera que, aplicando el Teorema 2.10, se sigue que para todo $C \subseteq \aleph_{1}$, existe $c \subseteq \omega$ tal que $C=\left\{\alpha<\aleph_{1}:\left|A_{\alpha} \cap c\right|<\aleph_{0}\right\}^{4}$. Por otro lado, el conjunto ternario de Cantor es un subconjunto de $\mathbb{R}$ cerrado y no contable que además es homeomorfo al producto de contables copias de $\{0,1\}$, es decir, $\{0,1\}^{\omega}=2^{\omega}$ con la topología producto. Así que, sin pérdida de generalidad, se puede suponer que $\mathcal{A}$ es un subconjunto de un conjunto cerrado de reales. Se afirma que todo subconjunto de $\mathcal{A}$ es un $F_{\sigma}$. En efecto, sean $\mathcal{B} \subseteq \mathcal{A}$ y $C:=\left\{\alpha: A_{\alpha} \in \mathcal{B}\right\}$. Por tanto, existe $c \subseteq \omega$ tal que $C=\left\{\alpha:\left|A_{\alpha} \cap c\right|<\aleph_{0}\right\}$, y en consecuencia, $\mathcal{B}=\left\{A_{\alpha}:\left|A_{\alpha} \cap c\right|<\aleph_{0}\right\}$. Ahora bien, para cada subconjunto finito $F$ de $\omega$ se define $c_{F}:=\{R \subseteq \omega: R \cap c=F\}$. Obsérvese que

$$
\mathcal{B}=\bigcup_{\substack{F \subseteq \omega \\ \text { F finito }}}\left(\mathcal{A} \cap c_{F}\right)
$$

y esta unión es contable, ya que la colección de subconjuntos finitos de $\omega$ es contable. De manera que basta con demostrar que cada $c_{F}$ es cerrado. Sin embargo, interpretando a $c_{F}$ como un subconjunto de $2^{\omega}$, esto se sigue fácilmente, pues un producto de cerrados es cerrado en la topología producto. Finalmente, se concluye que $c_{F}$ es cerrado, por tanto $\mathcal{B}$ es un $F_{\sigma}$ y en consecuencia $\mathcal{A}$ es un $Q$-set.

\footnotetext{
${ }^{4}$ Donde el Teorema 2.10 se aplica a $\mathcal{A}$ y $\mathcal{B}=\left\{A_{\alpha} \in \mathcal{A}: \alpha \in C\right\} \subseteq \mathcal{A}$.
} 


\section{Espacio de Moore derivado}

Definición 4.8. Sea $A \subseteq \mathbb{R}$ no contable. El espacio de Moore derivado de $A, M(A)$, se define como $\mathbb{R}_{+}^{2} \cup A^{5}$, dotado con la topología cuyos abiertos son los abiertos usuales de $\mathbb{R}_{+}^{2}$ y los conjuntos de la forma $\{a\} \cup D$, donde $a \in A$ y $D$ es el interior de un disco en $\mathbb{R}_{+}^{2}$ tangente al eje $x$ en $a$. A este tipo de abiertos se los denomina burbuja en $a$.

A continuación se estudian algunas propiedades básicas del espacio de Moore derivado, en particular se demuestra que, efectivamente, es un espacio de Moore:

Teorema 4.9. Sea $A \subseteq \mathbb{R}$ no contable. Entonces $M(A)$ es $T_{1}$, separable, de Moore y no metrizable, y $A$ es discreto como subespacio de $M(A)$.

Demostración. Sea $A \subseteq \mathbb{R}$ no contable. Que $M(A)$ es $T_{1}$ y regular es inmediato. Es separable ya que $\left\{(p, q) \in M(A): p \in \mathbb{Q}\right.$ y $\left.q \in \mathbb{Q}^{+}\right\} \subseteq M(A)$ es contable y denso en $M(A)$. Por otro lado, si para todo $n \in \omega$, se define

$$
\mathcal{U}_{n}:=\left\{B(x, 1 / n): x \in \mathbb{R}_{+}^{2}\right\} \cup\left\{\{x\} \cup D_{x}(1 / n): x \in A\right\},
$$

donde cada $D_{x}(1 / n)$ es el interior de un disco de radio $1 / n$ tangente a $x$, entonces es claro que $\left\{\mathcal{U}_{n}\right\}_{n \in \omega}$ es un desarrollo sobre $M(A)$. Para demostrar que $A$ es discreto en $M(A)$ es suficiente con notar que para todo $a \in A,\{a\}=(\{a\} \cup D) \cap A$. Finalmente, razonando por contradicción, si $M(A)$ es métrico, como también es separable, entonces es segundo contable, y por tanto es Jones-compacto; sin embargo, esto es absurdo, ya que $A \subseteq M(A)$ es no contable y discreto, por lo que no tiene puntos de acumulación. Luego $M(A)$ no es metrizable.

En consecuencia, cuando $A$ es no contable, entonces $M(A)$ es un espacio separable y de Moore, que no es metrizable. De manera que solo resta determinar condiciones para que el espacio de Moore derivado sea normal. Los siguientes resultados establecen dichas condiciones:

Lema 4.10. Si $A$ es un $Q$-set y $Y \subseteq A$, entonces $Y$ y $A \backslash Y$ se pueden separar con abiertos disjuntos en $M(A)$.

Demostración. Sean $A$ un $Q$-set y $Y \subseteq A$. Como $A$ es discreto en $M(A)$, entonces $Y$ y $A \backslash Y$ son cerrados, y por tanto existen colecciones contables de subconjuntos cerrados de $\mathbb{R},\left\{F_{n}\right\}_{n \in \omega}$ y $\left\{G_{n}\right\}_{n \in \omega}$, tales que

$$
Y=A \cap \bigcup_{n \in \omega} F_{n} \quad \text { y } \quad A \backslash Y=A \cap \bigcup_{n \in \omega} G_{n} .
$$

Sea $n \in \omega$. Nótese que $Y \cap G_{n}=\emptyset$, y por tanto, si $y \in Y$, la distancia Euclidiana entre $y$ y $G_{n}$ es positiva. Así que, como para cada $x \in A \cap G_{n}$ existe una burbuja en $x, B_{x}^{n}$, entonces con un cómputo sencillo se puede construir, para cada $y \in Y$, una burbuja en $y$, $B_{y}$, tal que para todo $x \in A \cap G_{n}$, se tiene que $B_{y} \cap \overline{B_{x}^{n}}=\emptyset$. De manera que si se define

$$
U_{n}:=\bigcup_{x \in A \cap G_{n}} B_{x}^{n},
$$

\footnotetext{
${ }^{5}$ Donde $\mathbb{R}_{+}^{2}$ denota el semiplano superior sin el eje $x$. Además identificamos la recta real con el eje $x$.
}

[Revista Integración, temas de matemáticas 
entonces $A \cap G_{n} \subseteq U_{n}$ y $\overline{U_{n}} \cap Y=\emptyset$. Análogamente, se puede construir un abierto $V_{n}$ tal que $A \cap F_{n} \subseteq V_{n}$ y $\overline{V_{n}} \cap(A \backslash Y)=\emptyset$.

Como consecuencia de lo anterior, existen colecciones de abiertos en $M(A),\left\{U_{n}\right\}_{n \in \omega} \mathrm{y}$ $\left\{V_{n}\right\}_{n \in \omega}$, tales que $A \backslash Y \subseteq \bigcup_{n \in \omega} U_{n}, Y \subseteq \bigcup_{n \in \omega} V_{n}$ y para todo $n \in \omega$ se satisface que $Y \cap \overline{U_{n}}=\emptyset$ y $(A \backslash Y) \cap \overline{V_{n}}=\emptyset$. Ahora bien, para todo $n \in \omega$, se definen los abiertos

$$
W_{n}:=U_{n} \backslash \bigcup_{i=0}^{n} \overline{V_{i}} \text { y } Z_{n}:=V_{n} \backslash \bigcup_{i=0}^{n} \overline{U_{i}},
$$

y se consideran

$$
W:=\bigcup_{n \in \omega} W_{n} \quad \text { y } Z:=\bigcup_{n \in \omega} Z_{n} .
$$

Es claro que $W$ y $Z$ son abiertos en $M(A)$ tales que $A \backslash Y \subseteq W, Y \subseteq Z$, y además, por la construcción, es claro que son disjuntos. Finalmente $W$ y $Z$ son una separación en abiertos disjuntos de $Y$ y $A \backslash Y^{6}$.

Teorema 4.11 (R. H. Bing, 1951). Si $A$ es un $Q$-set, entonces $M(A)$ es normal.

Demostración. Sean $A$ un $Q$-set, $F$ cerrado y $G$ abierto en $M(A)$ tales que $F \subseteq G$. Se definen $F^{+}:=F \cap \mathbb{R}_{+}^{2}$ y $G^{+}:=G \cap \mathbb{R}_{+}^{2}$. Nótese que $F^{+}$es cerrado en $\mathbb{R}_{+}^{2}$ y por tanto, como el plano superior es normal, existe un abierto $H_{1}$ tal que $F^{+} \subseteq H_{1} \subseteq{\overline{H_{1}}}^{E} \subseteq G^{+}$, donde ${\overline{H_{1}}}^{E}$ es la clausura euclidiana. Ahora bien, sea $Y:=A \cap F$, es decir, $Y$ es la colección de puntos de $A$ que están en $F$. Por el lema anterior, $Y$ y $A \backslash Y$ pueden separarse con abiertos disjuntos. Así que, existe un abierto $U$, tal que $A \subseteq U, U \cap(A \backslash Y)=\emptyset$ y además, sin pérdida de generalidad, se puede suponer que

$$
U=\bigcup_{x \in A \cap F} V_{x}
$$

donde para cada $x \in A \cap F, V_{x}$ es una burbuja en $x$. Consideremos $U_{x}$ para cada $x \in A \cap F$, como una burbuja en $x$ tal que $U_{x} \subseteq V_{x}$ y $\overline{U_{x}} \subseteq G$. Finalmente, definiendo

$$
H:=H_{1} \cup \bigcup_{x \in A \cap F} U_{x}
$$

se tiene que $H$ es un abierto en $M(A)$ tal que $F \subseteq H \subseteq \bar{H} \subseteq G$ y por tanto, $M(A)$ es normal.

El recíproco del Teorema anterior es cierto: si $M(A)$ es normal, entonces $A$ es un $Q$-set (véase [6, p. 47]). Así que, en general, $M(A)$ no es normal, y por tanto es un ejemplo de un espacio de Moore que no es normal.

Corolario 4.12. La existencia de un Q-set implica la existencia de un espacio separable y normal de Moore no metrizable.

\footnotetext{
${ }^{6}$ Nótese que este razonamiento es similar al que se lleva a cabo usualmente para demostrar que todo espacio regular y de Lindelöf es normal.
}

Vol. 38, $\left.\mathrm{N}^{\circ} 1,2020\right]$ 
Ahora bien, con los resultados de esta sección se tiene lo siguiente: $\mathbf{M A}+\neg \mathbf{C H}$ implica la existencia de un $Q$-set, y por el Corolario anterior se sigue la existencia de un espacio normal y separable de Moore que no es metrizable, es decir, $\neg \mathbf{M W C}$. Así que teniendo en cuenta la consistencia de $\mathbf{M A}+\neg \mathbf{C H}$ y el Lema 2.1, se concluye:

Corolario 4.13. $\neg \boldsymbol{M W C}$ es consistente con $\boldsymbol{Z F C}$.

Finalmente, del Corolario 4.3 y del resultado anterior, se sigue el objetivo de este artículo:

Teorema 4.14. $\boldsymbol{M W C}$ es independiente en $\boldsymbol{Z F C}$.

\section{Agradecimientos}

Los autores ofrecen sus agradecimientos al jurado del artículo por sus acertadas observaciones y sugerencias, las cuales contribuyeron a mejorar el contenido y la presentación del documento inicial. También al profesor Carlos Enrique Mejía, quien se tomó el tiempo de leer el artículo y darnos su impresión y recomendaciones acerca del mismo.

Este artículo se obtiene como resultado del trabajo de grado en Matemáticas del estudiante Andrés Felipe Uribe Zapata, titulado Sobre la independencia de la hipótesis del espacio normal de Moore (véase [7]) realizado en la Universidad Nacional de Colombia, sede Medellín, durante el segundo semestre del año 2019. En dicho trabajo de grado se abordan, además de la independencia de $\mathbf{M W C}$, los aspectos lógicos y conjuntistas fundamentales del problema y algunos otros detalles sobre la conjetura del espacio normal de Moore, así como la relación que tiene con la existencia de cardinales medibles.

\section{Referencias}

[1] Bing R.H., "Metrization of topological spaces", Canad. J. Math. 3 (1951), 175-186.

[2] Fleissner W.G., "Normal nonmetrizable Moore space from continuum hypothesis or nonexistence of inner models with measurable cardinals", Proc. Nat. Acad. Sci. U.S.A. 79 (1982), No. 4, 1371-1372.

[3] Jones F.B., "Concerning normal and completely normal space", Bull. Am. Math. Soc. 47 (1937), 671-677.

[4] Kunen K., Set Theory: An introduction to independence proofs, North-Holland Publishing Co., Amsterdam, 1980.

[5] Nyikos P.J., "A provisional solution to the normal Moore space problem", Proc. Amer. Math. Soc. 78 (1980), No. 3, 429-435.

[6] Tall F.D., "Set-theoretic consistency results and topological theorems concerning the normal Moore space conjecture and related problems", Thesis (Ph. D), University of Wisconsin, Madison, 1969, $53 \mathrm{p}$.

[7] Uribe-Zapata Andrés F., "Sobre la independencia de la hipótesis del espacio normal de Moore", Trabajo de grado en Matemáticas, Universidad Nacional de Colombia, Medellín, 2019, 70 p.

[Revista Integración, temas de matemáticas 